\title{
Perry G. Wang and Weixuan (Eds): Hydrophilic Interaction Liquid Chromatography (HILIC) and Advanced Applications
}

Peter A. Sewell

Published online: 26 May 2012

(C) Springer-Verlag 2012

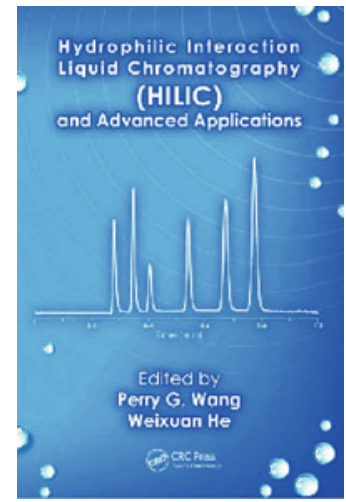

Bibliography

Hydrophilic Interaction Liquid

Chromatography (HILIC)

and Advanced Applications

Perry G. Wang and Weixuan (Eds)

CRC Press, Boca Raton, FL, USA 2011,

$\mathrm{pp} \mathrm{xx}+589$

ISBN 978-1439807538

GBP 108.00, USD 169.95

This book is No. 103 in the Chromatographic Science Series, a series of Textbooks and Reference books were edited by Nelu Grinberg and Founding Editor Jack Cazes. Previous authors in the series include such well-known names as J. Calvin Giddings, Lloyd R. Snyder, Walter G. Jennings, Phyllis R. Brown, Eli Grushka, Klaus Unger, Raymond. P. W. Scott, Joseph Sherma, and many others who have helped to develop chromatography into the most powerful separation technique available to the analytical chemist today.

HILIC, introduced by Dr. Andrew Alpert in 1990, is a variation of the earliest form of chromatography usually referred to as normal-phase chromatography which used a polar stationary phase and a non- (or less polar) mobile phase. In HILIC, organic solvents which are miscible with water are used with polar materials such as amino, cyano, diol, and silanol silica stationary phases. It is sometimes referred to as 'reverse reversed phase' or 'aqueous normal phase' chromatography.

P. A. Sewell ( $\square)$

Ormskirk, Lancashire, UK
The benefits of HILIC with high organic content mobile phases compared to other chromatographic techniques can be listed as:

- Lower back pressures and higher flow rates.

- Higher sensitivity for LC/MS analysis due to increased ionization.

- Improved peak shape and sensitivity giving more accurate quantitation of polar compounds such as peptides and nucleic acids.

- Retention of analytes that might not be retained by reversed phase chromatography.

- Capability of direct injection of samples following solid phase extraction (SPE) or liquid-liquid extraction, increasing throughput and facilitating sample preparation.

This book describes the new technology and provides detailed information for HILIC applications in the fields of environmental sciences, food analysis, clinical chemistry, pharmaceutical analysis and research, and biotechnology. The lack of a full understanding behind HILIC is acknowledged as is the availability of commercial HILIC columns. As in the early days of chromatography, the practice came before the understanding, and the range of applications described in this book is an invaluable source for the analytical chemist working in industry and in the research laboratory.

I cannot guarantee the accuracy of the analyses and their conclusions, but I am sure that the editors will have used due diligence in refereeing the papers, as I am sure the publisher has in proofing the manuscript. The print and diagrams are all very clear, making for easy reading. In over 600 pages, I would not be surprised to find a mistake, but this in no way would reduce the very high standard of publication. 